\title{
A NEW IDEA OF BIM SYSTEM FOR VISUALIZATION, WEB SHARING AND USING HUGE COMPLEX 3D MODELS FOR FACILITY MANAGEMENT.
}

\author{
F. Fassi a *, C. Achille a, A. Mandelli a, F. Rechichi a , S. Parri ${ }^{\text {b }}$ \\ a Politecnico di Milano, ABC Department, 20133 Milano, Italy \\ (francesco.fassi, cristiana.achille, alessandro.mandelli, fabrizio.rechichi@polimi.it) \\ b Newthread srl - parri@newthread.it \\ Commission V, WG V/4
}

KEY WORDS: BIM, 3D Visualization, 3D models, web sharing

\begin{abstract}
:
The work is the final part of a multi-year research project on the Milan Cathedral, which focused on the complete survey and threedimensional modeling of the Great Spire (Fassi et al., 2011) and the two altars in the transept. The main purpose of the job was to prepare support data for the maintenance operations involving the cathedral since 2009 and still in progress.

The research job had begun addressing our efforts to identify which methods would allow an expeditious but comprehensive measure of complex architectural structure as a whole. (Achille et al., 2012) The following research works were focused mainly to find an efficient method to visualize, use and share the realized 3D model.
\end{abstract}

\section{INTRODUCTION}

\subsection{General introduction}

The presented work will answer the final question asked by the paper presented to 4th ISPRS International Workshop, 3DARCH 2011: "3D Virtual Reconstruction and Visualization of Complex Architectures" Trento, Italy, 2-4 March 2011. (Fassi et al., 2011) The main topic of that job was about the best strategy to survey and model huge and complex architectures. The study case was the research activities conducted on the main spire of the Milan's Cathedral that have led to creation of a huge three-dimensional model of the spire characterized in great detail and high complexity. Initially the aim of the job was to build a 3D complete model of the spire in order to extract all feasible measures useful for the restoration site, but the feeling was that a complex and complete 3D model can give more. For this reason that job was concluded by asking the question: "A huge 3D model: what does it for?" (Fassi et al., 2011). The efforts of these last years were addressed in developing a sort of BIM system working on the web that would allow the use of the three-dimensional models during facility managements, supporting both the filed activities on the yard and the design and data updating in the technical offices (Fassi, 2012).

\subsection{The state of art}

In the last years we are witnessing a sudden development of. "Building Information Modeling" (BIM) system. It is "a digital representation of physical and functional characteristics of a facility. A BIM is a shared knowledge resource for information about a facility forming a reliable basis for decisions during its life-cycle" (NBIMS, 2012). This is only one of the many definitions of BIM but it makes clear in a few words the meaning and the potential of these systems. In fact in the recent years there has been international awareness that BIMs can make the design, construction and the different "building" operations more efficient, optimizing and speeding up building processes (Coates, 2010). Usually and until today, the Architecture, Engineering and Construction Industry is focusing only on new buildings without paying attention to the Cultural Building Heritage. So, the need to refurbish, but also all management and conservation processes of the existing buildings makes BIM for Cultural Heritage an essential research field in the last years (Del Giudice et al., 2013). The use of BIM can help in the understanding and preservation of heritage both for the professional and the general public.

Indeed it is exactly in the field of Cultural Heritage where 3DBIM systems have big potential, because the "CH objects" are expected to live forever and the maintenance processes, are continuous and endless into the future.

Moreover "A basic premise of BIM is collaboration by different stakeholders at different phases of the life cycle of a facility to insert, extract, update or modify information in the BIM to support and reflect the roles of that stakeholder" (NBIMS, 2010) and this is valid more than in other places, right in the world of $\mathrm{CH}$. In fact, all activities require collaborations across disciplines, technologies and sectors, such as history, archaeology, cultural studies, anthropology and other areas of humanities and social science.

The three main tasks are: i) object survey, ii) object representation and iii) visualization/sharing of information. Today the survey phase is relatively fast thanks to laser scanning and automatic image based algorithms. The creation of the $3 \mathrm{D}$ model is the real bottleneck of the whole workflow. Nowadays, the research is focusing mainly on this task and in particular developing algorithms able to speed up the modelling phase.

The main topic is normally the simplification of the modelled structures using parametric or semantic modelling procedures.

\footnotetext{
* Corresponding author. This is useful to know for communication with the appropriate person in cases with more than one author.
} 
These methodologies have achieved very good results in case of "regular simply study cases" but are inadequate for complex and irregular objects (as in the archaeological case of studies) or for big scale representation where simplification is not possible. Moreover commercial systems are not able to model complex freeform object in a real-based way and also the imported models can not be fully used in the "information system" as smart objects. For these reasons different types of volumetric modelling procedures, typical of Archicad or Revit, or standard parametric libraries (HBIM) make commercial systems inadequate or not sufficient for many $\mathrm{CH}$ case of studies.

The third objective faces the difficulty to share and make usable by different people a considerable amount of data. The main issue is the use and sharing of three-dimensional models that are normally of large dimension, both for the high resolution with which are represented and for their effective extension and complessity. Nowadays there are many webservices able to visualize and share 3D models. Normally they are little objects and little 3D models. In the field of Cultural Heritage there are few systems able to visualize complex and large 3D data on the web. One is Cyark viewer able to visualize high resolution pointcloud or mesh surfaces on a dedicated Webgl system directly on the browser (Silla L., 2012).

Another interesting system is Potree (Potree, 2014). It is a free WebGL based point cloud viewer for very large datasets. It is based on Scanopy project with the purpose to develop efficient data structures and algorithm to allow displaying huge pointbased models (Wimmer M. et al., 2006). These are very interesting and useful visualization systems. They can be very useful because all survey methods, both image and range based, produce quite huge and dense point clouds, that for many purposes, can be enough to represent the object without any futher elaborations. Another interesting project is 3DHOP (3D Heritage Online Presenter) developed by the Visual Computing Lab, ISTI-CNR. It is a collection of tools and templates for the creation of multimedia interactive Web presentations of digital cultural artifacts. It is a free software able to visualize online a $3 \mathrm{D}$ mesh model in the native Nexus format. It is thought for online visualization and museal presentation with the aim to be used by anyone with simple webpage-creation skills, without the need for specific Computer Graphic expertise. Also this application is built using the WebGL API technology and the SpiderGL JavaScript library. An interesting aspect is the possibility to costumize the html pages inserting every kind of textual information.

An important citation is due to the Nubes Project developed by CNRS (De Luca L., 2013); that is an information system at the architectural scale of representation. The main aim of that project is to create a web-system able to allow a multidisciplinary analysis of heritage building creating complex relations between geometric 3D representation of the building with heterogeneus data concerning various domains (technical, historical, documentary). It is the first complete example of information system sharing on the web created ad hoc for Cultural Heritage.

\subsection{The three main characteristics of the new system}

The system is designed from three main considerations.

The main first idea was to break away from the commercial software that nowadays are unsuitable for the multi-faceted world of Cultural Heritage. They are unable (totally or partially) to handle complex "real based modelled shapes", large scales modelled objects and big dimension areas or buildings. In addition, the main problem is the final use of the model. Commercially, the implemented applications are rarely, and only partially, interesting for a cultural heritage object, because they have been conceived for the design in the maintenance of new buildings. Moreover, they do not include features useful for maintenance operations and restoration activities. This is because "cultural heritage objects" are unique from the artistic, historical, architectural and constructive point of view: each has its own strong identity that is impossible to standardize.

The starting point is the realization of a highly customizable system able to easily adapt itself to the different cases allowing to work with different type of objects, different representation scale and resolutions, also simultaneously showing and sharing different type of information and managing different and unique maintenance operations.

For this reason, the presented system is able to visualize huge dimension and high numbers of different 3D models. They can be built with any external software and can be linear simple blocks (structure) or complex high-resolution multi-scale free form models (decorations) or both integrated together. That mean that:

- there are no constraints on the number of the viewable objects,

- there are no constraints on the level of detail of each model. So the users can visualize each object with different level of detail, depending on their needs,

- the users can place models of any type, regardless of the method or software by which they were created (manual modelling, parametric modelling or semantic modelling or real based modelling),

- the original $3 \mathrm{D}$ model format is preserved by the system (nurbs or mesh models) in order to let the modification in the original modelling software, but automatically managed/transformed as mesh (also textured) inside the system viewer.

The second key aspect is a consideration about the operators working in Cultural Heritage field. The modern explosion of 3D models means that even this world will have to create professional roles able to build, modify and update threedimensional virtual representation of an object. In general, however, the user and the classical operator in the field of cultural heritage will not have the knowledge to use, modify complex three-dimensional models requiring primarily dedicated skills and high-level hardware technologies. The second aim of the system is to solve this problem. The system should be easy to use and easy to learn even for non-experts. It should have a user-friendly interface and should be used with common devices. That is the only way to ensure that the system becomes integral part of the maintenance and restoration practices and very attractive to the variegated world of operators in $\mathrm{CH}$ field.

The third consideration is that a digital 3D model is expected to become the representation of the future. This is valid in any contemporary field of study, but can be extremely significant in the field of Cultural Heritage. A 3D model is a complete representation of the "object" that can be analysed and studied in its entirety, without any effort of abstraction by the operators. Digital 3D models add big chances in the process of knowledge, providing new possibilities on the way to study, manage, monitor, visualize, conserve and valorise a cultural heritage object. However, to achieve this purpose it should be dynamic and eternal, that means it should be forever upgradeable and modifiable in the future; it should be "for everybody", i.e. visualized and used easily by different operators and "from everywhere" that means that a digital 3D model should be shared and used in a collaborative way. 


\section{THE WEBBIM SYSTEM}

For these reasons, the presented system, named BIM3DSG, is thought divided in three parts:

1. a first part that is thought to be mainly used from professional and 3D specialist. It gives full access to the whole system and all functions inside the modelling software or standalone (work in progress). It is required to add or modify (export process) 3D models into the system; every kind of previously modelled surface are supported.

2. The second part is instead thought for all other users and, therefore, it is specially designed to be easy to use and usable in mobility on different devices, such as PC, notebook, tablet and smartphones. This part requires only a web browser and gives full access to the whole system and all the functions inside the web browser, except the ability to add or modify 3D models. It can also be used on device with few hardware resources because it permits to view objects at different levels of detail, computed automatically by the system during the export process, through approximation of the mesh surface extrapolated from the original model.

3. at last, the system needs a central database that collects both 3D models and all information and additional files related to each object. It is ready-made by system administration but can be easily automatically initialized by the user. The use of a central database allows users to share and synchronize all changes in real-time.

Regardless of whether using the first or the second part, BIM3DSG allows the user to:

- view all or part (selected through some search functions) of the objects previously exported in the system

- view automatically computed BIM information of each object, such as area, volume, position, ...

- add/edit/view user BIM information and files (photo, video, documents, $\mathrm{dwg}, \ldots$ ) associated with one or more objects

- add/edit/view maintenance, restoration and building site activities and also all related information and files (photo, video, documents, dvg, ...)

- $\quad$ view past situation, and also view past and present situation at the same time for comparison intent

In both parts powerful caching mechanisms have been implemented, in order to ensure the user-friendliness and the appeal of the system, allowing almost instantaneous loading time, even if the available connection is slow.

\subsection{Distinctive feature}

What makes our system possible is the innovative idea (patent pending, protocol number MI2014A002016) to split the whole model into each constitutive entities and save all of them separated one from other into a central database; in addition a series of search functions that also allow users to also load only portions of the whole model.

The type of constitutive entities is defined by the user, not only for each project, but also inside a project, to easily adapt to the widest range of needs. For example, if the whole threedimensional complex model is the representation of a cathedral, the single constitutive entity could be a capital of a column, the column itself, or even an entire spire, but also a constitutive part of the capital of the column.
The only requirement for the user is to use, within the modelling software, different objects for each constitutive entity; during the export process, our system can recognize each object and, automatically and transparently to the user, is responsible to divide each object in a separate file that will be stored into the central database.

When the user needs to view the model, he can search for it and load only the portions of interest through a series of search functions; e.g. the user can make a selection by:

- using one or more filters on identification parameters of the constitutive entities, such as for example the area, the zone, the sector, the type and name of the single element

- including of one or more, or ranges of unique codes, associated automatically by the system for each constitutive entities during the export process

- asking for a cross-section of the entire model (or an area of the entire model), with a height defined by the user and focused on a single element selected by the user

- $\quad$ asking for a selection of a ball around on the whole model (or an area of the entire model), with a radius defined by the user and focused on a single element selected by the user

Other queries can be included by request and can involve maintenance, restoration and building site activities.

The most interesting thing about the ability to load only the portions of interest of the whole model is that it allows users to use so defined model that couldn't be loaded entirely at full quality on the actual hardware, or that could be loaded, but with too poor performance.

In addition, the ability to use different levels of detail (automatically computed) on the web allows you to display always the entire model, even when it should not be possible to do it because of hardware limits, with the only limit of using a lower level of detail.

Thanks to this feature, our system allows the user to:

- $\quad$ insert and manage any number of different objects different from each other, even if they are not parametrizable;

- insert and manage any number of any resolution (chosen by the user) models of objects which geometry is not default;

- $\quad$ use simultaneously simple and complex objects.

\subsection{The central database}

The central database is rather installed on a remote server or on the cloud, in order to be accessible by a plurality of workstations and devices through the conventional Internet connection. However, if needed, the database can be both local or placed within a local network.

The central database stores not only the internal structure and the textual BIM information inserted by users, but also the models files (both in the format used by modelling software and JSON format, automatically computed and used for web visualization), the BIM files inserted by users and any other necessary data and files.

We chose to use PostgreSQL on Linux, because is Open Source and has a lot of useful features. However, our system can be adapted to use other database, for example Microsoft SQL Server or Oracle Database. 


\subsection{The modelling management part}

We wrote a complex plug-in for Rhinoceros, that gives users access to all functions and features, except the use of level of detail, that is distinctive for web visualization.

The choice of Rhinoceros derived by the fact that it is one of the few software that support both NURBS and MESH, and it is easy to use and learn.

Our plug-in is written in C\# and .NET. For the UI, we used Windows Form in the first version, while in second version we are using WPF embedded inside Windows Form (embedded because Rhinoceros is written in Windows Form), because WPF is more attractive and modern.

Our plug-in also uses ITinnovationsLibrary (Rechichi, 2014) and only in second version Xceed WPF Toolkit and a modded version of AvalonDock.

The export process is written with high multithread parallelism, so that it can use all physical and virtual core available; it can reduce the time of export process to about $7 / 8$ times on consumer processor and at least 11/12 times on even more on high performance workstation processor.

We have planned for the future to release a stand-alone application that can export OBJ files into the system. We can also develop, on request, plug-ins for other modelling software.

\subsection{The online platform}

The ability to use a common web browser to view 3D models and perform all tasks, except the export process, is a very important feature of our system.

First, it helps to persuade common user towards the use of the system during the daily work. Then it can be also used in mobility and on mobile devices and it is cross-platform. Last but not the least, there is no additional cost for customers because browsers are free, and one organization (for example a public administration) could give read-only access (full access permission and separation, based on UNIX like schema, are supported by BIM3DSG) to models and some data to everyone for free without any cost.

The touch-screen support makes BIM3DSG even more simple to use and let it also run on touch-screen only device, e.g. in a museum where we can have a touch display for visitors.

BIM3DSG, for visualizing 3D models inside a web browser, uses WebGL through SceneJS library. The core system uses also Javascript, JQuery, php, css. The web interface is based on JQuery EasyUI.

\subsection{Modules}

The main functionality of BIM3DSG can be summarized into a block diagram, as we can see in Figure 1.

The first step is the user authentication: BIM3DSG allows the management of advanced user permissions (users and groups), allowing to prevent or to allow access and/or modification of each single element and each single file inserted, according to a UNIX like access scheme.

Through the export process user can add the constitutive entities and/or modify all the previously inserted entities (only inside modelling management part).

Then we have the import process, by which at any time the user can import and load all or part of the previous inserted entities, in order to view, analyze, modify, or access all the BIM information and data associated with them.

Once the user has imported the entities, it is possible to access a lot of feature for viewing and entering data, information and files on each single constitutive entities and its model, starting from the selection of the same. BIM3DSG let user to:

- insert any type of textual information

- insert multimedia files (photo, video, ...) of any format

- insert other files of any format (documents, reports, pdf, dwg, ...)

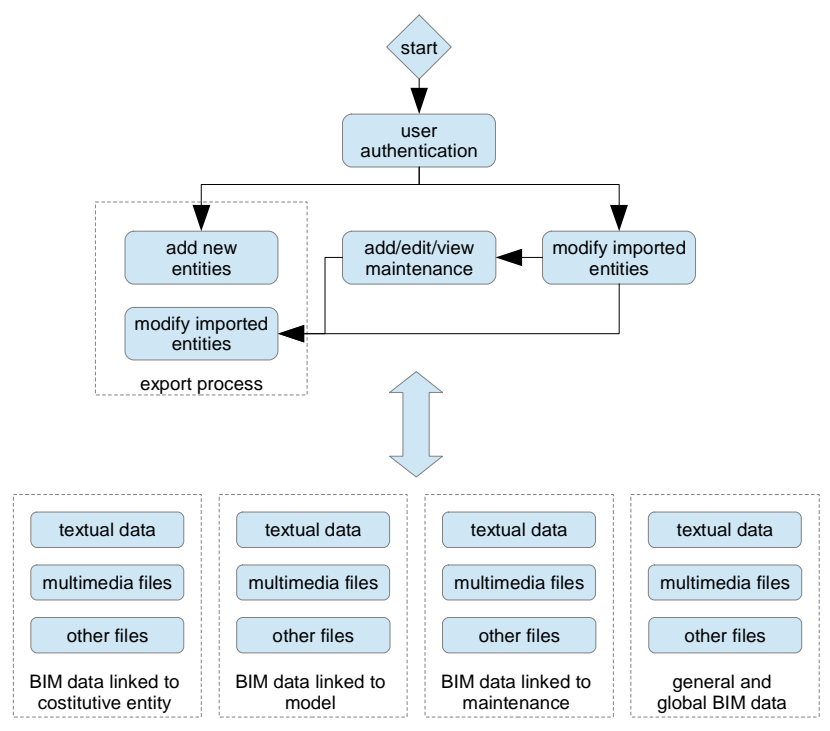

Figure 1. block schema for the main features of BIM3DSG.

Each information can be mapped on both a single constitutive entity, or on its model, or on the maintenance events, or as well on a group of them.

Furthermore, user can view some automatically computed BIM information of each model, such as area, volume, position, ... These data are computed by BIM3DGS during the export process.

Eventually the user can add/edit/view maintenance, restoration and building site events, starting as usual from the selection of constitutive entities involved.

\subsection{The export process}

The export process is the core of our system, therefore we go on analizing it in more detail.

First, BIM3DSG validates the objects selected for exportation process and write permission; then it displays them to the user, allowing the identification of new objects and modifying objects (identification can be both manual or automatic).

After this first step, BIM3DGS makes a final validation of the previous choice and finally start the actual export process, that is mainly composed from the following step:

1. extraction of identification parameters, such as area, zone, sector, type (from the layer structure) and name

2. saving one temporary file for each constitutive entities to export; each file contains one and only one constitutive entity and it use the specific format of used application (for example $3 \mathrm{dm}$ for Rhinoceros); after the system completes the step and the step below, it start to upload the temporary files into the database

3. pre-inizialization of the database for the new entities and cleaning of some records for the modified entities, saving a backup (all user information remains 
untouched); it also locks all items to export to avoid conflicts during web visualization from other user

4. a multithread process, where on threads do some operation on one entity, including:

- extraction of the mesh, if object is a mesh, or comupute it from NURBS

- computation of volume, area, bounding box, its center and its radius, ...; volume and area are computed only for high quality LoD (Level of Detal)

- conversion of the model in JSON format, saving it in one or more temporary file, which eventually include also UV texture map coordinates for applying the texture

- updating database informations and datas; JSON files are upload in background just were each thread is completed

5. if there are other LoD to create, the system starts a multithread elaboration for decreasing the size of each object: the system uses a own algorithm particularly designed for decimating mesh (described below). When the system completes this multithread process backs to four step

6. when all process and file uploads are completed, the system removes the access lock previously inserted and ends its operations

Our algorithm for mesh decimation permit to approximate it on a grid of increasing size (LoD1 $>1 \mathrm{~mm}$, LoD2 $>$ 2mm, Lod3 $>4 \mathrm{~mm}$, and so on), by rounding off vertex coordinates on the grid and merging with the same resulting coordinates; our algorithm removes faces collapsed in one line or point and recalculates the normals. It manages UV texture coordinates in the same way of vertexes. Acting in this way, the mesh is reduced in size, but keeps its properties (e.g. if we start from a closed mesh, reduced mesh is also closed).

\subsection{BIM3DSG v2 preview}

BIM3DSG v2 is currently in development, even if some features are already ready.

For BIM3DSG we rewrite almost all of the code, with a better structure to make it even faster than before; in addittion, also thanks to the decision to adopt WPF for the UI and some framework for WPF, we have focused on giving the software a more visual appeal and easier usability. This could seem a low priority task, but today usability, rapidity and attractive looking makes the difference between a very useful system, but never or rarely used, and a system that spreads out and starts to be used for the daily work.

With regards to the capabilities, we moved mainly toward two directions, both are designed for an use as general as possible of the system: texture support and the ability to change dynamically the information fields.

Texture support is in fact required in some cases of study, where having only the model of the structure is not enough, but also visual information about cover or decoration is required. Texture are now supported both in the modelling management part and the online platform.

Regarding the ability to dynamically change the information fields, it is a very important feature because allows the system to be used in several different situations and fields, adapting it to the user request and need, without the requirement to rewrite the code.
Now we are working on another important feature, the ability to share some information between two or more entities, so that the user can write or modify at the same time all common information to a group of entities (e.g. material information, or survey technique).

\section{THE WEBBIM FOR FACILITY MANAGEMENT}

All objects belonging to Cultural Heritage have a particular and unique characterization. Also about intervention and maintenance, it is quite impossible to standardize methodologies and techniques because the possible activities on the objects could be countless and also conducted in a very different way according to the different operators and the condition of the object at that particular time of its life. An information system should be able to transform itself in order to fit with the infinite different situations.

The presented system is built with this purpose. An operator can quite easily modify the database from time to time adding ad hoc workings, proper information fields and parameters tables. In this way many restoration intervention can be easily presented and compiled as textual information using easy dynamic tables linked to the objects as textual list of executed processes with the addition of any associated parameters.

In many cases this is not enough.

The restoration or maintenance activities imply the modification of the structure or part of it. Textual information are inadequate to describe the operations and specially they can not represent the geometric changes of the objects. A Building Information System should be able to support a series of ad hoc created intervention on the object that contemplate the complete replacement or a partial modification of a piece or multiple pieces from a geometric point of view. Moreover, the system is able to connect all the modifications with ad hoc created "father-son" relationship and providing in this way the possibility to estract in real time the modification history of each object, visualizing also its 3D geometric modification.

\subsection{The case of the Milan Cathedral.}

The case of study of the Milan Cathedral is a very good example of those concepts. Some dedicated features are specially created in the presented Web Information System to manage the ordinary and extraordinary maintenance operated by the Veneranda Fabbrica.

\subsubsection{The survey and the model construction.}

The survey has been conducted with a multi-scale and multidata approach. Multi-data means using various measurement sensors, depending on the necessity and used individually or simultaneously. The multi-scale concept, instead, is the integration of objects detected in different scales for different purposes or needs in the model. The use of different technologies is imposed by different factors characterizing the Spire, such as the material, the survey complexity due to the restricted spaces or to the element elevation, to the cable-stayed scaffolding and to the presence of the restoration site.

The most used technique is the photogrammetry, both as regards the construction of simpler and more linear parts of the structure (image modeling), and as the decorated parts (image matching). The choice to use the photogrammetry as the main survey method has been made to direct the research toward a low cost process. Besides the geometric survey, it has also been led a photographic survey describing every single element of the steeple, pointing out the conservation status and the small details hard to model and to be represented graphically. 
The model is built inside Rhinoceros and guarantees a measurements extraction at 1:50 scale. Many details are modelled at bigger scale in order not to lose in the representation important shapes and geometries testimonials of the constructive characterization of the Milan's Cathedral. For this reason many decorative parts are modelled at 1:5 scale (Fassi et al. 2011; Achille et al 2012).

\subsubsection{The centuries-old tradition of the cathedral facility} management.

It is quite impossible to describe in few words the traditional methods to maintain the cathedral by Veneranda Fabbrica. Synthesizing at most, one can say that the main task of the Fabbrica is to guarantee the structural safety of the cathedral. As said by the Architect of the Veneranda Fabbrica, Benigno Moerlin Visconti Castiglione, the Milan's Cathedral is a living monument and is experienced by people, it has to be safe and functional. It is built entirely in Candoglia marble that undergoes over the years strong structural weakening due to rain washout and especially to air pollution. (Fassi et al., 2011) So all the cathedral is is monitored by careful periodic inspections, from which all maintenance activities are designined. Normally the main interventions operations consist in substitution, in whole or in part, of the degraded marble blocks, anchorages and reinforcement structures. Cleaning and restoration operations are normally scheduled for decorations and statuary. In this way the building site of the Milan Cathedral is an eternal construction site that regularly intervenes to heal those parts that threaten its stability and its security.

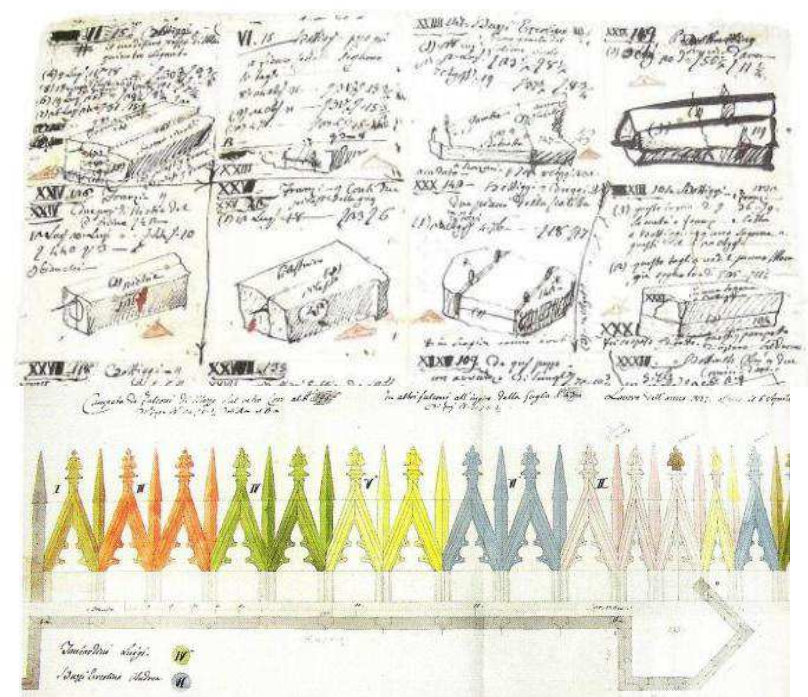

Figure 2. Old 3D drawings of single marble blocks processing with related info and description. 3D representations and colours are used to better represent and describe the management activities. The new BIM will support the activities respecting the old classic way of work

3.1.3 The new facility management system. In the system, several dedicated features were developed to support the old traditional activities of the Veneranda Fabbrica concerning both ordinary and extraordinary maintenance activities on the Milan's Cathedral and to document all working phases and all restoring activities. The same $3 \mathrm{D}$ model was built specifically for this purpose, by modeling each marble block and creating a "big LEGO" of pieces which focus on all the maintenance activities.
Developing this part particular attention was dedicated to create a system able:

1. to manage fast geometric changes in the structures,

2. to increase the real time communication between jard and offices increasing the automatic data updating,

3. to increase the portability of the system in order to encourage its use at the construction site.

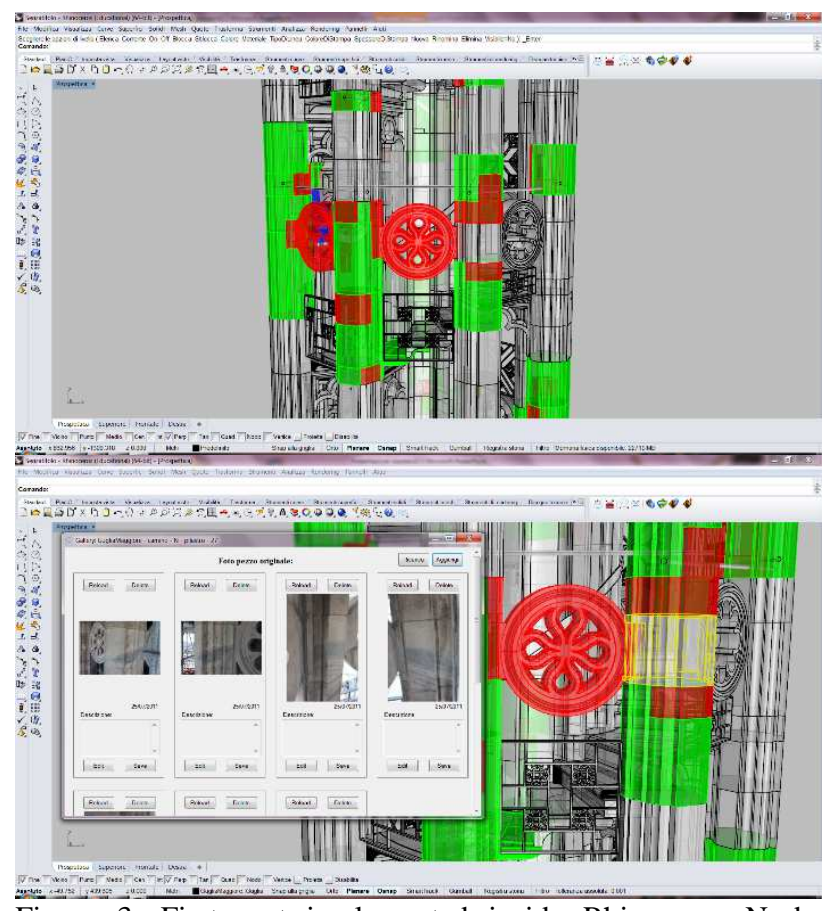

Figure 3. First part implemented inside Rhinoceros Nurbs Modeller. It is possible to see 3D models with different colour in function of different restoration activities. Info and images can be loaded and visualized with ad hoc panel. 3D model and info are loaded from the external $\mathrm{DB}$ and can be used without saving them locally

The system lets to:

- enter any maintenance activities with detailed description (text or images) that does not change the structure of the model previously entered,

- insert any kind of maintenance that alter the structure of the model, with either a one to one, or in a one to many, or in a many to one, or in a many to many relationship,

- manage and visualize maintenance objects with different colours in function of the intervention typologies, state of progress, time and history of multi interventions,

- manage and visualize text information and images and other files linked to the intervention,

- manage and visualize text information and images and other files linked to the blocks interested by the intervention,

- apply query or selection in function of intervention typology, time and positions,

- load past situation so users can go back and forth in the history of the building structure, and also load past and actual situations at the same time for comparison intent

- associate any management information to the interventions, such as the assignment of a given job to 
an user, planning assistance, recording the hours and costs required or incurred for interventions etc,

- $\quad$ estimate the weight of objects when it is known that the density of the material (weight is automatically computed from the volume); it is also possible to automatically estimate the cost of any replacement if it is known the price of the raw material and its density,

- combine all the information, images and files linked to the single object to an entire area or region or sector or object type,

- connect full resolution models (1:1 scale) for 3D printing and industrial automatic construction.

All the features can be used by both systems (web and desktop), and all changes are always synchronized in real time. All features are usable concurrently from multiple locations and multiple users, both locally and remotely, through the Internet, although, for obvious reasons, some editing operations require a temporary write-lock of the involved objects, in order to prevent concurrency hazards.
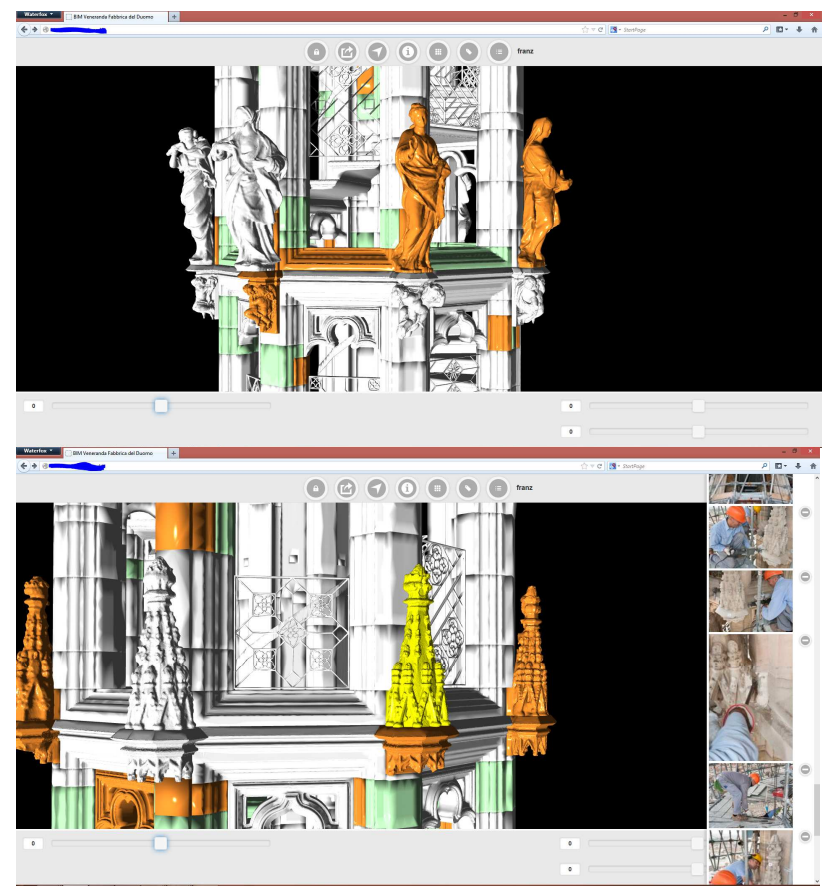

Figure 4. Web Viewer with the model loaded in LOD 1

\section{CONCLUSION}

The presented system answers the initial question. It has the triple goal to:

1. visualize huge and complex $3 \mathrm{D}$ models on the web. It is quite similar to others online viewer, but more flexible in terms of visualizable 3D model types and more powerful in terms of visualized object dimensions,

2. share information and any kind of data (textual, image video, etc.) linked to the model in a simply and immediate way using the web,

3. keep track of the changes of the objects in the years.
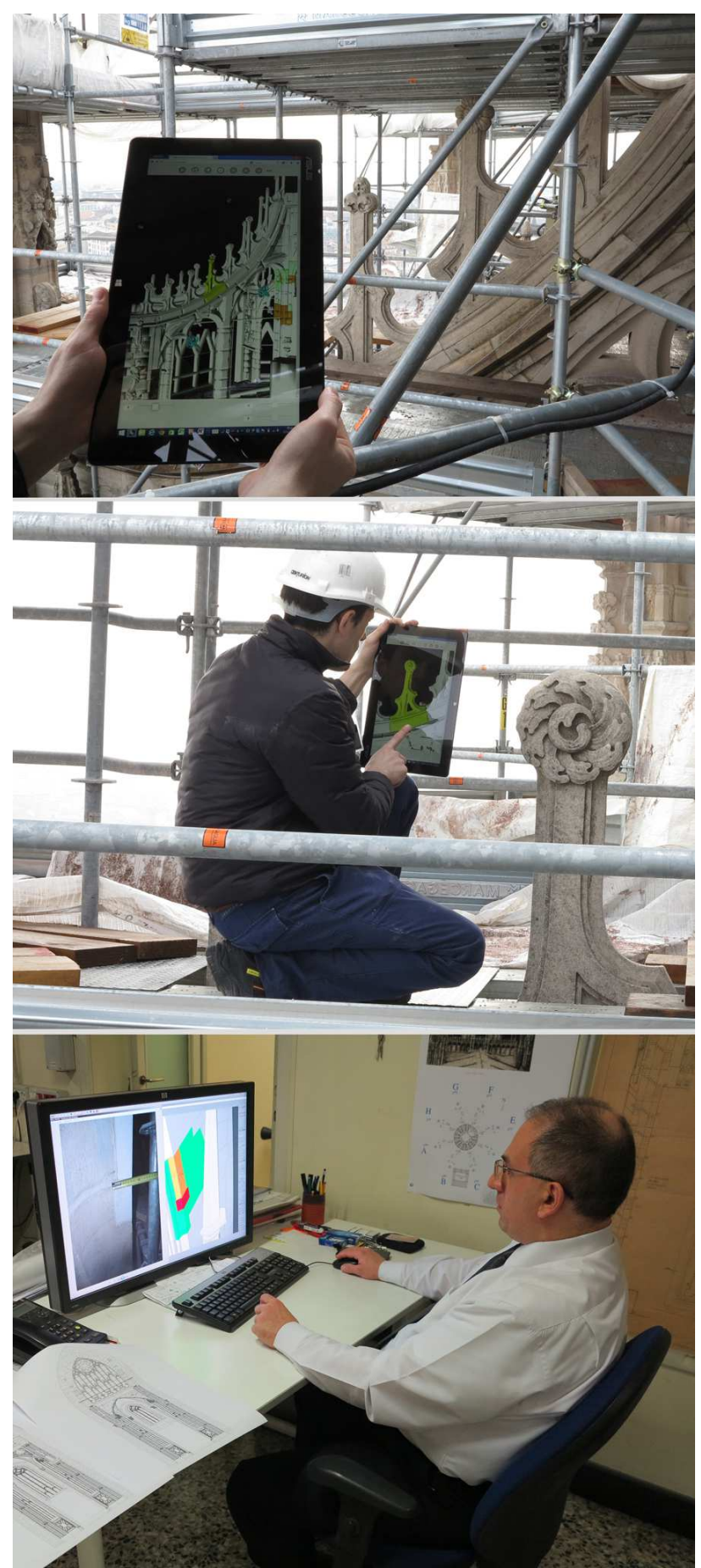

Figure 5. Example of daily use of our system for maintenance activities of the Milan Cathedral: (5.1)(5.2) yard operation which requires the insertion of a new maintenance event using the web interface on a tablet; (5.3) a 3D specialistic is updating, in his office, 3d models using photos, information and datas just inserted from operator in fig 5.1 e 5.2

It is important to underline some key aspects that make the system useful and attractive for different stakeholders in the world of Cultural Heritage:

- "the ease-of-use of the system", that can be used by the end user without the aid of any software with fee;

- the data enter and consulting is simple and intuitive; 
- ad hoc choice of the modelling software, even if requires some preparation and exercise, i.e. the end user doesn't need to have a dedicated modeling competence.

It is currently used daily by the Veneranda Fabbrica of Duomo both during the yard activities and in the technical office. The first year of testing gave good results in terms of time saving in designing and scheduling operations. The real time updating of the "drawings" of the cathedral and the real time computations of expenses are particularly successful. The use of the system as method of presentation of the performed works and results was appreciated because immediate and always available on the web. Smaller success but still recognized by the users is the usage of the system during construction activities. In fact, the need of continuous internet connection is the weak point of the system. The study of solutions to this problem is already planned in the future developments.

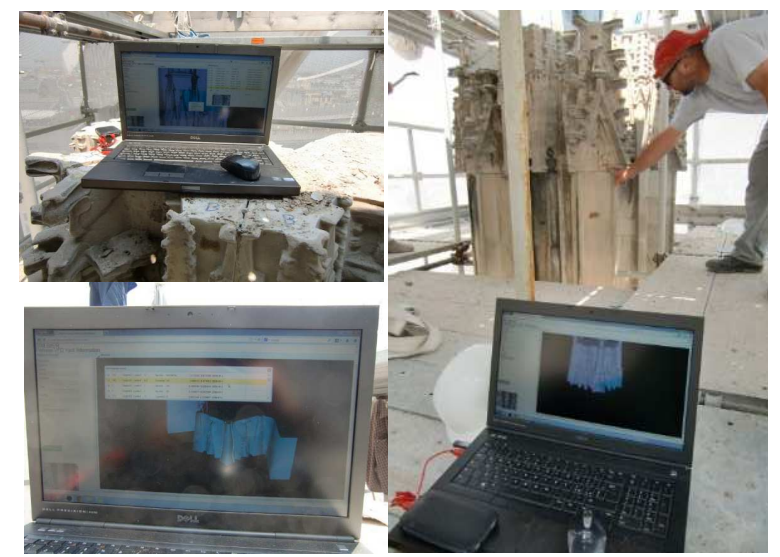

Figure 6. The use of the WEB Bim system in the yard as support for the disassembly of the oldest spire of the Milan Cathedral.

The remarkable interest for this project has propelled the idea of improving and engineering the system and especially adapting it in order to be used by other actors. Tests, further research and developments are in progress to make it even more customizable and suitable for general architectonic and archaeological case studies.

\section{ACKNOWLEDGEMENTS}

This work was supported by Veneranda Fabbrica of Milan's Cathedral. Special thanks to the director Eng. Benigno Moerlin Visconti Castiglione for his support and availability. And to all staff especially Francesco Aquilano and Massimiliano Regis.

\section{REFERENCES}

Achille, C., Fassi, F., Fregonese L. 2012, 4 Years History: from 2D to BIM for $\mathrm{CH}$. The main spire on Milan Cathedral. In: $G$. Guidi, A. C. Addison. Proceeding of VSMM 2012. Virtual Systems in the Information Society. Milan, 02/09/2012 05/09/2012, p. 377-382, IEEE, ISBN: 9781467325622

Coates, P., Arayici, Y., Koskela, L., Kagioglou, M., Usher, C., O'Reilly, K., 2010, he key performance indicators of the BIM implementation process, ICCBE 2010, Jun 30 2010, Nottingham

Cyarc, http://www.cyark.org/

De Luca, L., 2013, 3D Modelling and Semantic Enrichment in Cultural Heritage, 54th Photogrammetric Week in Stuttgart. September 9-13.

Del Giudice, M., Osello, A., 2013, Bim for Cultural Heritage, Int. Arch. Photogramm. Remote Sens. Spatial Inf. Sci., XL5/W2, 225-229, doi:10.5194/isprsarchives-XL-5-W2-225-2013, 2013.

Fassi, F., Achille, C., Gaudio, F., Fregonese, L., 2011, Integrated strategies for the modelling very large and complex architectures, Int. Arch. Photogramm. Remote Sens. Spatial Inf. Sci., XXXVIII-5/W16, 105-112, doi:10.5194/isprsarchivesXXXVIII-5-W16-105-2011, 2011.

Fassi, F., Achille, C., Fregonese, L., 2011, Surveying and modelling the Main Spire of Milan Cathedral using multiple data sources. The Photogrammetric Record, 26: 462-487. doi: 10.1111/j.1477-9730.2011.00658.x

Fassi, F., Parri S., 2012, Complex Architecture in 3D: From Survey to Web. International Journal of Heritage in Digital Era, vol. 1, p. 379-398, ISSN: 2047-4970

Potree, 2014, Online at: http://potree.org/wp/ (accessed: 25 January 2015)

Rechichi, F., 2014, ITinnovations Library, online http://www.itinnovations.it

Silla, L., 2012, CyArk Releases New Online 3D Viewer Online at: http://www.cyark.org/news/cyark-releases-new-online-3dviewer

NBIMS, 2012. National Building Information Model Standard Project Committee, http://www.buildingsmartalliance.org/index.php/nbims/faq/ (accessed: 2 March 2012).

NBIMS, 2010. National Building Information Modeling Standard, online http://www.wbdg.org/pdfs/NBIMSv1_p1.pdf

Wimmer, M., Scheiblauer, C., 2006, Instant Points. In Proceedings Symposium on Point-Based Graphics 2006, pages 129-136. July 2006.

3Dhop, 2014, Online at: http://vcg.isti.cnr.it/3dhop/index.php

WebGL (2013), online https://www.khronos.org/webgl/

SceneJS (2010), online http://scenejs.org

Xceed WPF Toolkit (2010), online http://wpftoolkit.codeplex.com

AvalonDock (2007), online https://avalondock.codeplex.com

JQuery EasyUI (2010), online http://www.jeasyui.com 